\title{
ON THE SUM OF TWO BOREL SETS
}

\author{
P. ERDÖS AND A. H. STONE ${ }^{1}$
}

Abstract. It is shown that the linear sum of two Borel subsets of the real line need not be Borel, even if one of them is compact and the other is $G_{\delta}$. This result is extended to a fairly wide class of connected topological groups.

1. Introduction. If $C$ and $D$ are Borel subsets of the real line $R$, need $C+D$ be Borel ${ }^{2}$ Here $C+D$ denotes the set $\{x+y \mid x \in C, y \in D\}$. In the simplest cases the answer is obviously "yes"; for example if at least one of $C, D$ is countable or open, or if both are $F_{\sigma}$ sets. We shall show that in the next simplest case, in which $C$ is compact and $D$ is $G_{\delta}$, the answer is "no"; $C+D$ need not be Borel. ${ }^{3}$ (It will, of course, be analytic; in fact the sum of two analytic sets is analytic, being a continuous image of their product.)

The answer to the corresponding question about the plane (with + denoting vector sum) has been known for some time, though it does not appear to be in the literature. The present construction imitates the plane counterexample in the space $A \times B$, where $A, B$ are suitable additive subgroups of $R$, and then transfers it to $A+B \subset R$. The axiom of choice is not required.

2. The subgroups. As was shown by von Neumann [3], if we put

(1) $f(x)=\sum_{n=1}^{\infty} p(p([n x])) / p\left(p\left(n^{2}\right)\right)$, where $p(a)=2^{a}$, then the numbers $f(x), x>0$, are algebraically independent. Clearly $f$ is strictly increasing, and is continuous at each irrational $x$; hence, if $P^{+}$denotes the set of positive irrationals, $f\left(P^{+}\right)$is homeomorphic to $P^{+}$and therefore contains a Cantor set $K{ }^{4}$ In turn, $K$ clearly contains two (in fact, $c$ ) disjoint Cantor sets $K_{1}, K_{2}$. We let $A, B$ denote the additive subgroups of $R$ generated by $K_{1}, K_{2}$ respectively. Thus

Received by the editors July 22, 1969.

AMS Subject Classifications. Primary 2810; Secondary 2635, 5401, 2210.

Key Words and Phrases. Borel set, analytic set, complete metric spaoe, Cantor set, algebraically independent, connected topological group, absolute $G_{\delta}$.

1 The second author's contribution was supported by the National Science Foundation.

${ }^{2}$ We are indebted to Mr. B. V. Rao for calling our attention to this problem.

${ }^{3}$ A closely related result has been obtained independently, by a different method, by C. A. Rogers [4].

"By "Cantor set" we mean any space homeomorphic to the usual Cantor ternary set; that is, a compact, zero-dimensional dense-in-itself metric space. In particular, the Cantor subsets of $R$ are just the nonempty bounded perfect nowhere dense sets. 
(2) $A$ and $B$ are $\sigma$-compact and contain Cantor sets, and

$$
A \cap B=\{0\} .
$$

\section{The sets.}

Theorem. There exist a Cantor set $C \subset R$, and a $G_{\delta}$ subset $D$ of $R$, such that $C+D$ is not Borel.

Proof. The subgroup $A$ contains $K_{1}$ which contains a homeomorph $P_{1}$ of the space of irrational numbers. Take a non-Borel analytic subset $E$ of the Cantor set $K_{2}$ (cf. [1, p. 368]). There is a continuous map $g$ of $P_{1}$ onto $E$; let $G$ be its graph, a subset of $P_{1} \times K_{2} \subset A \times B$. As in [1, pp. 366, 367], $G$ is closed in $P_{1} \times B$; and $P_{1}$ is an absolute $G_{\delta}$. Thus $G$ is $G_{\delta}$ in $A \times B$, and therefore

$(A \times B) \backslash G$ is $\sigma$-compact.

Let $F=A \times\{0\}$. Note that $F+G$ (where + here refers to the group operation in the direct product $A \times B)$ is not Borel in $A \times B$, because its intersection with $\{0\} \times B$ is the non-Borel set $\pi_{2}(G)=E$.

Now consider the homomorphism $\phi: A \times B \rightarrow R$ given by $\phi(a, b)$ $=a+b$. Clearly $\phi$ is continuous and (by choice of $A$ and $B$ ) one-toone. We note that $\phi(F+G)$ is not Borel in $R$, since otherwise the continuity of $\phi$ would show that $\phi^{-1}(\phi(F+G))$ would be Borel in $A \times B$; but this set is $F+G$. Thus

(4) $\phi(F)+\phi(G)$ is not Borel in $R$.

We have, however,

(5) $\phi(F)=A=\bigcup_{m=1}^{\infty} A_{m}$ where each $A_{m}$ is a Cantor set.

For we may take $A_{m}=$ set of all numbers of the form $a_{1}+a_{2}+\ldots$ $+a_{m}$ where $\pm a_{i} \in K_{1}(i=1,2, \cdots, m)$. This is a Cantor set because it is clearly compact and perfect, and also nowhere dense (since otherwise $A=R$, contradicting (4)).

Again, $\phi(G)$ is $G_{\delta}$ in $A+B$, for (since $\phi$ is 1-1) its complement $(A+B) \backslash \phi(G)$ is the image under $\phi$ of $(A \times B) \backslash G$, and is therefore $\sigma$-compact, by (3). But $A+B$ is $F_{\sigma}$ in $R$; hence $\phi(G)$ is $G_{\delta \sigma}$ in $R$, and we may write $\phi(G)=\bigcup_{n=1}^{\infty} G_{n}$ where each $G_{n}$ is a $G_{\delta}$ in $R$. Now (4) and (5) show that $\bigcup_{m, n}\left(A_{m}+G_{n}\right)$ is non-Borel; hence there exist $m, n$ such that $A_{m}+G_{n}$ is non-Borel, and we merely take $C=A_{m}, D=G_{n}$.

4. Remarks. Mr. Rao has called to our attention that, starting from the above theorem, L. A. Rubel's method [5] will produce pathological Borel measurable functions on the real line. For instance, if $\phi(x)=\sup _{-\infty<t<\infty}|f(x+t)-f(x-t)|$, then the Borel measurability of $f$ does not imply that of $\phi$. 
It may also be worth remarking that not every analytic subset of $R$ is expressible as the sum of two (or more) Borel sets. For example, if $H$ is an arbitrary non-Borel analytic subset of $[0,1]$, and $L=H \cup\{3\}$, then $L$ is not expressible in the form $X+Y$ for any nondegenerate sets $X, Y$. For otherwise it is easy to see that, for some $\lambda \neq 0, L \cap(L+\lambda)$ contains a translate of $X$ (take $\lambda=y_{1}-y_{2}$ where $y_{1}$, $y_{2} \in Y$ ), and thus that diam $X<1$. Similarly diam $Y<1$ and so diam $(X+Y)<2$, contradicting $X+Y=L$.

5. More general groups. Mycielski [2] has generalized von Neumann's construction, showing in particular that every connected topological group with a complete metric, which is either locally compact or abelian, contains an independent Cantor subset. The foregoing arguments apply virtually unchanged ${ }^{5}$ to show that every such group (written additively) contains two Borel sets (in fact a compact set and a $G_{\delta}$ ) whose sum is not Borel. It would be interesting to know whether this remains true if "connected" is weakened to "nondiscrete".

\section{REFERENCES}

1. C. Kuratowski, Topologie. Vol. 1, 2nd ed., Monografie Mat., vol. 20, PWN, Warsaw, 1948; English transl., Academic Press, New York and PWN, Warsaw, 1966. MR 10, 389.

2. J. Mycielski, Independent sets in topological algebras, Fund. Math. 55 (1964), 139-147. MR $30 \# 3855$.

3. J. von Neumann, Ein System algebraisch unabhängiger Zahlen, Math. Ann. 99 (1928), 134-141.

4. C. A. Rogers, A linear Borel set whose difference set is not a Borel set, Bull. London Math. Soc. (to appear).

5. L. A. Rubel, A pathological Lebesgue-measurable function, J. London Math. Soc. 38 (1963), 1-4. MR 26 \#5123.

Hungarian Academy of Sciences, Budapest, Hungary and University of Rochester, Rochester, New York 14627

${ }^{5}$ In the nonabelian case, $A+B$ need not be a group, and $\phi$ need not be a homomorphism; however, we still have $\phi(F+G)=\phi(F)+\phi(G)$ because of the special nature of $F$. 